\title{
BMJ Open Guided and unguided internet-based vestibular rehabilitation versus usual care for dizzy adults of 50 years and older: a protocol for a three-armed randomised trial
}

\author{
Vincent A van Vugt, ${ }^{1}$ Johannes C van der Wouden, ${ }^{1}$ Judith $\mathrm{E}$ Bosmans, ${ }^{2}$ \\ Martin Smalbrugge, ${ }^{1}$ Willianne van Diest, ${ }^{1}$ Rosie Essery, ${ }^{3}$ Lucy Yardley, ${ }^{3}$ \\ Henriëtte E van der Horst, ${ }^{1}$ Otto R Maarsingh ${ }^{1}$
}

To cite: van Vugt VA, van der Wouden JC, Bosmans JE, et al. Guided and unguided internet-based vestibular rehabilitation versus usual care for dizzy adults of 50 years and older: a protocol for a three-armed randomised trial. BMJ Open 2017:7:e015479.

doi:10.1136/bmjopen-2016015479

- Prepublication history for this paper is available online. To view these files please visit the journal online (http://dx.doi.org/10.1136/ bmjopen-2016-015479).

Received 13 December 2016 Accepted 21 December 2016

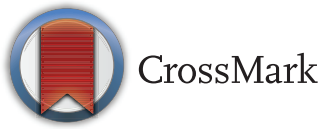

${ }^{1}$ Department of General Practice \& Elderly Care Medicine, Amsterdam Public Health research institute, VU University Medical Center, Amsterdam, The Netherlands ${ }^{2}$ Department of Health Sciences, Faculty of Earth and Life Sciences,

Amsterdam Public Health research institute, Vrije Universiteit Amsterdam, Amsterdam, The Netherlands ${ }^{3}$ Department of Psychology, University of Southampton, Southampton, UK

Correspondence to Vincent A van Vugt; v.vanvugt@vumc.nl

\section{ABSTRACT}

Introduction: Dizziness is a common symptom in general practice with a high prevalence among older adults. The most common cause of dizziness in general practice is peripheral vestibular disease. Vestibular rehabilitation (VR) is a safe and effective treatment for peripheral vestibular disease that entails specific exercises to maximise the central nervous system compensation for the effects of vestibular pathology. An internet-based VR intervention has recently been shown to be safe and effective. Online interventions are low cost and easily accessible, but prone to attrition and non-adherence. A combination of online and face-to-face therapy, known as blended care, may balance these advantages and disadvantages.

Methods and analysis: A single-blind, three-arm, randomised controlled trial among patients aged 50 years and over presenting with dizziness of vestibular origin in general practice will be performed. In this study, we will compare the clinical and costeffectiveness of stand-alone internet-based VR and internet-based VR with physiotherapeutic support ('blended care') with usual care during 6 months of follow-up. We will use a translated Dutch version of a British online VR intervention. Randomisation will be stratified by dizziness severity. The primary outcome measure is the Vertigo Symptoms Scale-Short Form. Intention-to-treat analysis will be performed, adjusting for confounders. The economic evaluation will be conducted from a societal perspective. We will perform an additional analysis on the data to identify predictors of successful treatment in the same population to develop a clinical decision rule for general practitioners.

Ethics and dissemination: The ethical committee of the VU University Medical Center approved ethics and dissemination of the study protocol. The insights and results of this study will be widely disseminated through international peer-reviewed journals and conference presentations.

Trial registration number: Pre-results, NTR5712.

\section{Strength and limitations of this study}

- This is a protocol for the first randomised controlled trial in general practice that investigates the effectiveness of the combination of online vestibular rehabilitation (VR) and face-to-face therapy (blended care) as a form of treatment for patients with dizziness of vestibular origin.

- By using a three-arm design, we will investigate the effectiveness and cost-effectiveness of stand-alone internet-based VR and blended care compared to usual care during 6 months of follow-up.

- We will attempt to develop a clinical decision rule for predictors of successful treatment to ensure personalised treatment. This will allow general practitioners to discriminate between patients who should receive stand-alone internetbased VR and those who are more likely to benefit from a blended care approach.

- Our internet-based intervention was designed for patients aged 50 years and over with access to the internet. Owing to these requirements, the treatment might not be accessible to all adult patients with dizziness of vestibular origin.

\section{INTRODUCTION}

Dizziness is a common symptom in general practice. ${ }^{1}$ Among adults, the life-time prevalence of dizziness has been reported to be $20-30 \% .^{2-4}$ The prevalence of dizziness increases with age; in adults over 85 years old, the prevalence exceeds $50 \% .^{5}$ Dizziness is associated with a lower quality of life, occupational consequences and substantial interference with daily activities. ${ }^{6}$ It is a major risk factor for falling, which can be especially harmful for older adults who are prone to fractures. ${ }^{7}$ Owing to a decrease in work productivity and the high costs of medical care, 
dizziness also constitutes a substantial economic burden. ${ }^{4}$

The most common cause of dizziness in general practice is peripheral vestibular disease (33-38\%), which includes benign paroxysmal positional vertigo (BPPV), vestibular neuronitis and Ménière's disease (MD). Other causes of dizziness are cardiovascular diseases (7-18\%), neurological disorders $(10-15 \%)$ and psychiatric disorders $(1-17 \%) .^{8-10}$ In the Netherlands, as well as in the UK and the USA, $>90 \%$ of all patients with dizziness are treated by their general practitioner (GP) without any involvement of a specialist, ${ }^{9-11}$ mainly by reassurance, advice and symptomatic drug prescriptions. ${ }^{11}$ However, none of the commonly prescribed drugs for vertigo have a well-established curative or preventive effect. ${ }^{12}$

According to a recent Cochrane review-based on 39 randomised controlled trials (RCTs) involving 2441 participants-there is moderate-to-strong evidence that vestibular rehabilitation (VR) is a safe, effective treatment for peripheral vestibular disease. ${ }^{13} \mathrm{VR}$ entails that the patient carries out graded exercises for 10-20 min daily for 6-12 weeks. These exercises include specific eye, head and body movements that stimulate the vestibular system and promote neurological adaptation to the altered input from the damaged labyrinth. ${ }^{14} 15$ Performing these exercises may also help patients to overcome fear and avoidance of activities that provoke dizziness, and to regain skill and confidence in balance. ${ }^{14} 16$ Importantly, when examining the 39 RCTs mentioned above, ${ }^{13}$ most RCTs were performed among a selected population (eg, participants with canal paresis, otolith disorder or acoustic neuroma resection) and were conducted in a secondary/tertiary care setting. Only 2 of the 39 RCTs included a population representative of patients seen in primary care, these showed that nurse-delivered VR and booklet-based VR effectively reduce dizziness. ${ }^{1417}$

Despite the evidence for the effectiveness of VR, a previous survey suggests that $<3 \%$ of eligible primary care patients with dizziness ever receive VR. ${ }^{17}$ This may be due to a lack of availability or access to VR or a lack of knowledge about VR by the GP. ${ }^{11}$ To date, access to VR usually involves a costly referral process to secondary care. $^{18}$ Also, finding suitable therapists can be difficult. ${ }^{14}$ Prior to this proposal, we performed a survey among a sample of 1169 Dutch GPs to investigate the current use of VR in general practice. ${ }^{19}$ Of the 426 responding GPs, $93 \%$ reported that they never used VR-by themselves or by referral-for the treatment of patients with dizziness. Not knowing how to perform the technique was the most mentioned reason by GPs not to use VR $(92.4 \%)$. 'Takes too much time to execute' $(7.2 \%)$, 'doubts about the effectiveness' $(6.3 \%)$ and 'not recommended by national guidelines' $(4.5 \%)$ were other reasons mentioned by GPs for not using VR. Consequently, it is not only important to generate additional evidence for the effectiveness of VR in general practice, but also to consider alternative models of treatment delivery to support the GP.
In the past, VR has been successfully delivered via a booklet-based self-management programme. Bookletbased VR has been tested in two large primary care trials $^{1417}$ and a voluntary population of members of the Meniere's Society. ${ }^{20}$ All trials showed that application of $\mathrm{VR}$ resulted in a significant reduction of dizziness symptoms without any serious adverse events. ${ }^{14} 17 \quad 20$ The internet could prove to be an alternative method to reach patients with dizziness in an inexpensive, easily accessible way. Booklet-based treatments are perfectly suited to be transformed into internet-based interventions. An online intervention can be tailored to the patients' individual needs, featured audio-visual content and provided various means of motivational support (eg, discussion boards or personalised text messages) ${ }^{21}$ Internet use by older adults is growing rapidly. According to Statistics Netherlands (CBS), $80 \%$ of adults aged 65-75 years reported internet use in 2013, which is more than twice as much as in $2005 .^{22}$ A recent review by Aalbers $e t a l^{23}$ showed that internet interventions targeting lifestyle change in adults aged above 50 years can be effective. The authors concluded that internet-mediated interventions hold great potential in implementing effective lifestyle programmes and are capable of reaching large populations of older persons at low costs. ${ }^{23}$ With these advantages in mind, an online VR intervention based on the VR booklet was developed in the UK. ${ }^{18}$ The internet-based VR intervention has recently been compared to usual care in an RCT (Geraghty et al personal communication). The trial was conducted in a general practice population and included 296 patients. Patients in the online VR intervention group experienced significantly less dizziness and dizziness-related disability at 6 months, compared to those in the usual care group. We will translate the British online VR intervention into Dutch. No alterations in the content of the translated programme will be made.

In addition to this, we will also investigate a different form of delivery in which the use of the online intervention is supported by a healthcare professional. Despite the advantages, unguided internet interventions can be associated with substantial attrition and non-adherence. A combination of online and face-to-face therapy ('blended care') may reduce attrition and nonadherence, offering patients the best of both worlds. ${ }^{24-26}$ In one of the booklet-based VR trials, ${ }^{14}$ a subset of patients received additional telephone support by a trained vestibular therapist while performing the booklet self-managed programme. By focusing on addressing patient concerns, reassuring, encouraging adherence and ensuring proper use of the booklet, these patients demonstrated higher adherence to the treatment and a significant reduction in anxiety and depression. Face-to-face contact is likely to be even more effective than telephone support. In the Netherlands, physiotherapists with experience in vestibular therapy often make house calls, and they are used to encourage and 
reassure their patients when performing physical exercises. As such, they are perfectly suited to be the 'face' of an online VR intervention.

The principal aim of this research project is to investigate the effectiveness and cost-effectiveness of stand-alone internet-based VR and internet-based VR with physiotherapeutic support (blended care) for patients with dizziness aged 50 years and over in general practice in comparison with usual primary care. The online intervention that will be applied has been proved to be safe and effective for patients aged 50 years and over (Geraghty et al personal communication). By assessing the benefits of blended care, we may be able to improve the treatment of older patients with dizziness. Furthermore, it will offer GPs a new-immediate, easily accessible and inexpensive-clinical tool for treating dizziness in general practice. Additionally, we aim to develop a prediction rule to help GPs to assess which patient groups are best suited for each type of treatment.

\section{METHODS AND ANALYSIS}

\section{Study design}

We will perform a single-blind, three-arm, RCT among patients aged 50 years and older presenting with dizziness in general practice. We will compare the clinical and cost-effectiveness of stand-alone internet-based VR and internet-based VR with physiotherapeutic support ('blended care') with usual care. The intervention period will last 6 weeks and patients will be followed up at 3 and 6 months. The primary outcome measure will be dizziness symptoms at 6 months as measured by the Vertigo Symptom Scale-Short Form (VSS-SF). Figure 1 represents a flow chart of the study with an overview of the group classification and randomisation. By using the LifeGuide software, we will automatically (and nonintrusively) collect data on all aspects of intervention use, including which sections and pages are viewed, how often and for how long. In addition to this, we will perform semistructured interviews with 10-15 participants from trial arm 1 (stand-alone internet-based VR) and 10-15 participants from trial arm 2 (internet-based VR with physiotherapeutic support) to assess their experiences with the online intervention.

\section{Recruitment of study participants}

The large majority of patients will be recruited from the Academic Network of General Practices of the VU University Medical Center (ANH-VUmc). The ANHVUmc includes a total of 50 active GPs. The network should be a sufficient source to attain our inclusion number. Other general practices in the Netherlands (primarily in and around Amsterdam) may be approached if inclusion numbers from the ANH-VUmc prove to be insufficient. Eligible patients will be identified by means of a validated search strategy ${ }^{27}$ that selects from the electronic medical records those patients within the set age range who visited their GP because of dizziness in the past 2 years. The GP will screen the selected lists of patients for potential exclusion criteria or other pertinent reasons for not inviting them, such as a terminal disease or a severe psychiatric disorder. The identified and screened potential participants will be sent a package by their GP containing a letter, a brochure with information about the trial and a contact form. If a patient is willing to participate, he/she can contact the research assistant by phone, email or by returning the addressed contact form. On making contact, the research assistant will provide additional information if necessary and check the eligibility criteria. These criteria are a good command of the Dutch language, access to the internet and an email account, persisting dizziness at time of inclusion that has been present for at least 1 month, and that is exacerbated by performing head movements. If the participant is eligible, the research assistant will make an appointment for a visit at the participant's home. The research assistant will bring an informed consent form with an addressed return envelope. The participant will be able to ask questions about the trial and the informed consent form during this house visit. After the house visit, the participant will be given 1-2 weeks of time to consider if he/she wants to participate in the trial. During that time period, the research assistant will be available to answer any questions over the phone that might arise. To participate, the individual is required to sign the informed consent form and return it to the research team by using the addressed return envelope. After this signed informed consent form has been received by the research team, an email will be sent to the participant with a link to the intervention website.

\section{Sample size and power calculation}

The sample size calculation for our trial is based on the comparison between participants allocated to trial arm 1 (stand-alone internet-based VR) and those allocated to trial arm 3 (usual care). In Yardley et $a l \mathrm{~s}^{14}$ recent VR trial with VSS-SF as a primary outcome measure, booklet-based VR alone, compared with routine care, showed an effect size (Cohen's d) of 0.45, favouring booklet-based VR. Assuming that stand-alone internet-based VR will produce the same effect size as booklet-based VR alone, we will need 80 patients per group to test a two-tailed hypothesis with $\alpha=0.05$ and $\beta=0.20$. As attrition from internet-based interventions can be substantial, ${ }^{25}$ we will recruit a minimum of 100 participants per group (300 total sample) to allow for up to $20 \%$ attrition $(80 \times 100 / 80=100)$.

\section{Eligibility criteria}

Eligible patients are required to:

- be 50 years or older,

- possess a good command of the Dutch language,

- have access to internet and an email account,

- have consulted a GP with dizziness symptoms in the last 2 years, 


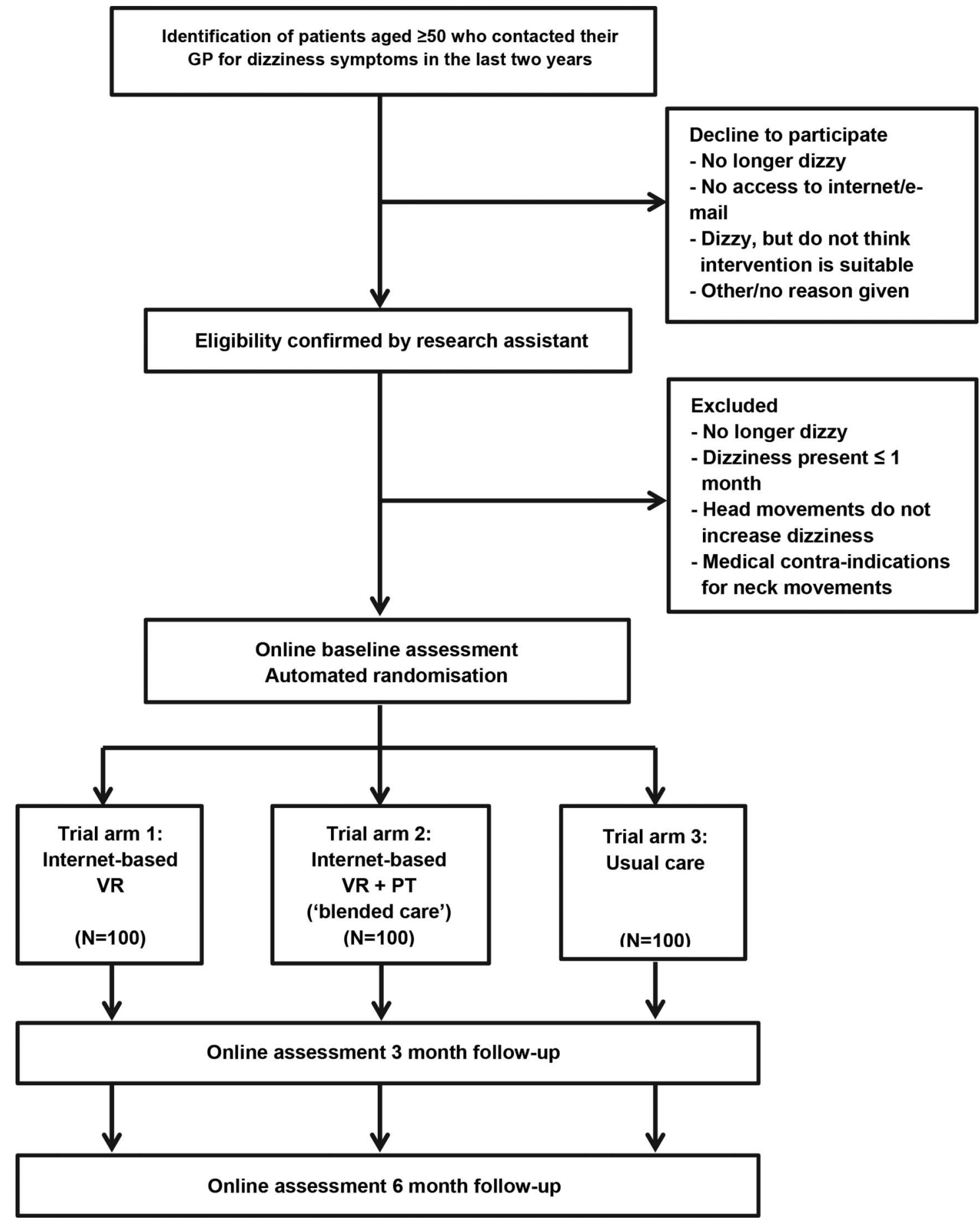

Figure 1 Flow-chart study. PT, physiotherapy; VR, vestibular rehabilitation.

- still experience dizziness at the time of inclusion, which has to have been present for at least a month and

- experience dizziness exacerbated by head movements; otherwise, the dizziness is unlikely to have a vestibular origin. ${ }^{14}$

The cut-off point for duration at 1 month was chosen because of acute BPPV; the Epley manoeuvre is deemed superior to VR. After 1 month, both treatments are considered equally effective. ${ }^{28}$ Reasons for exclusion are: an identifiable non-vestibular cause of dizziness in the electronic record of the patient; medical contraindications for making the required head movements (eg, severe cervical arthrosis); serious comorbid conditions that preclude participation in an exercise programme or current enrolment in another-interfering-study.

\section{Randomisation and blinding}

All patients are required to complete baseline measures online. The online software programme will randomly assign participants to one of the three arms. During the automated randomisation, patients will be stratified by 
dizziness severity according to their score on the VSS-SF. Participants with a VSS-SF score of $<12$ points will be classified as low severity patients, whereas participants with 12 or more points will be classified as high severity patients. Patients are automatically informed about their allocated group. When patients are allocated to trial arm 2 (blended care), an email will be sent to the principal investigator. The principal investigator will select and contact one of the trained physiotherapists, depending on the location of the patient. The physiotherapist will contact the patient within 24 hours to plan the first therapeutic session. Since VR is a behavioural intervention, it is not possible for patients to be blinded to allocation. At 3 and 6 months, they will receive an email-and, if necessary, a reminder-to complete follow-up measures online. If participants still fail to respond, an independent research assistant will call them to encourage them to fill out the questionnaires online. The research assistant analysing the questionnaire data will be blind to treatment allocation.

\section{Intervention}

\section{Internet-based VR (trial arm 1)}

VR entails specific exercises with the aim of maximising central nervous system compensation for vestibular pathology. Recently, specific components have been defined, namely compensation (using motion to habituate or reduce responsiveness to repetitive stimuli and to rebalance tonic activity within the vestibular nuclei), adaptation (using repetitive and provocative movements of the head and/or eyes to reduce error and restore vestibule-ocular reflex gain), substitution (promoting the use of individual or combinations of sensory inputs) and motor learning principles (changing movement behaviour). Additionally, deliberately provoking dizziness in a controlled context functions as a form of exposure-based behaviour therapy. ${ }^{15}{ }^{18}$ A VR booklet was developed by following principles of behaviour change, derived from cognitive-behaviour therapy and self-regulation theory. ${ }^{14}$ Self-efficacy is stimulated in the intervention by graded goal setting. This VR booklet was validated in three large trials..$^{14} 1720$ The content of the booklet has been the basis for a recently developed stand-alone internet-based VR intervention. This intervention was developed by Dr Lucy Yardley, ${ }^{18}$ member of our research group and coauthor of this manuscript. A prototype version of the intervention was tested among 18 adults in the UK. ${ }^{29}$ Participants were positive regarding how easy to navigate, visually appealing and informative they found the intervention. Think-aloud sessions provided valuable data for informing small amendments to further enhance acceptability of the intervention for target users. ${ }^{29}$ The recently conducted large trial by Geraghty et al $(\mathrm{n}=296)$ (Geraghty et al personal communication) has shown that the British version of internet-based VR improves dizziness and reduces dizziness-based disability in older primary care patients. Patients in trial arm 1 will get access to a careful Dutch translation of this stand-alone VR intervention.

The intervention period will last 6 weeks for every patient. In the first session, information about dizziness and a rationale for the VR exercises will be provided. These exercises represent the core of the intervention. The weekly 'timed exercise scoring test' (TEST) will also be explained in the first session. VR exercises will be personalised for the patient and take into account his/her symptoms and balance capabilities. The online TEST is used to tailor the set of exercises. All of the exercises will have written instructions with video demonstrations, each performed by a male and a female model. In the first session, all six exercises (see box 1) are performed sitting down, afterwards the patients are asked to score how dizzy each respective exercise made them feel. The intervention uses these scores to automatically produce an exercise prescription for the coming week, custom built to the patient's dizziness symptoms. When performing the exercises while sitting no longer causes dizziness symptoms, the patient will be asked to perform the exercises standing up. By using this approach, patients will gradually increase the intensity of their exercises. Patients are asked to perform these exercises two times per day for $10 \mathrm{~min}$. Patients will complete a different online session every week in the 6-week intervention period.

The intervention incorporates information and advice on behavioural coping strategies. Previous research showed that symptom control strategies led to significant subjective health improvement and reduction of dizziness-related impairment. ${ }^{20}$ Progressive muscle

\section{Box 1 Exercises}

1. Shake: Turn your head from right to left and back again 10 times in $10 \mathrm{~s}$. Twist your head round as far as it will go comfortably when you do this, and look in the direction your head is pointing. Wait $10 \mathrm{~s}$ after you have performed 10 complete turns, and then do 10 more.

2. Nod: Nod your head up and down and back again 10 times in $10 \mathrm{~s}$. Tip your head as far as it will go comfortably when you do this, and look in the direction your head is pointing. Wait $10 \mathrm{~s}$ after you have performed 10 complete turns, and then do 10 more.

3. Shake, eyes closed: Carry out the shake exercise with your eyes closed. Wait $10 \mathrm{~s}$ after you have performed 10 complete turns, and then do 10 more.

4. Nod, eyes closed: Carry out the nod exercise with your eyes closed. Wait $10 \mathrm{~s}$ after you have performed 10 complete turns, and then do 10 more.

5. Shake/stare: Hold your finger pointing upwards in front of you and carry out the shake exercise while staring at your finger. Do not let your eyes move from your finger. Wait $10 \mathrm{~s}$ after you have performed 10 complete turns, and then do 10 more.

6. Nod/stare: Hold your finger pointing sideward in front of you and carry out the nod exercise while staring at your finger. Do not let your eyes move from your finger. Wait $10 \mathrm{~s}$ after you have performed 10 complete turns, and then do 10 more. 
relaxation and breathing techniques can decrease dizziness-associated psychophysiological arousal, whereas cognitive restructuring (challenging negative dizzinessrelated thoughts) and problem solving may lessen dizziness-provoking anxiety. ${ }^{30}$ In addition, the internet intervention includes particular features to increase engagement. Scientific studies supporting the effectiveness of VR are summarised in plain language to increase patients' expectations regarding the intervention content. Email messages will be used to remind patients to $\log$ in to the intervention each week. Since our intervention is aimed at older adults, the look and design of the internet-based VR programme is simple with large font sizes and use of bullet points instead of paragraphs wherever possible. Importantly, internetbased VR will be offered to patients in addition to their usual GP care (ie, the intervention does not replace usual GP care).

\section{Internet-based VR and physical therapy}

(trial arm 2-blended care)

Patients in trial arm $2(n=100)$ will receive the same intervention as the patients in trial arm $1(n=100)$. This internet-based VR intervention is described in detail in the previous paragraph. In addition to the internetbased VR intervention, patients in trial arm 2 will receive two sessions by a trained physiotherapist. Although the advantages of a stand-alone internetbased intervention are numerous (eg, easily accessible, inexpensive, enormous reach and automated tailoring), unguided internet interventions can be jeopardised by substantial attrition (ie, a patient leaves the treatment before the treatment goals are achieved) and nonadherence (ie, a patient remains enrolled, but fails to attend the scheduled treatment). Previous research on depression showed that combining online sessions and regular face-to-face psychotherapy can increase adherence and effectiveness. ${ }^{24-26}$ Patients allocated to trial arm 2 will receive such blended care. The physiotherapeutic sessions will occur in week 1 and 3 of the 6-week intervention period and last for $45 \mathrm{~min}$ each. During these sessions, the physiotherapist will (1) provide information about the background of dizziness and VR; (2) elicit and address doubts and concerns about dizziness and VR; (3) teach the patient how to use the online intervention; (4) describe and take the patient through a set of VR exercises; (5) advise on how to anticipate and cope with obstacles to adherence and (6) provide support and encourage adherence. The first session will focus on making the patient feel comfortable with the VR exercises. The second session will focus more on adherence to the treatment and will therefore be planned in the third week. Performing VR exercises can slightly increase the dizziness at first. During the second session, the physiotherapist will reassure the patient of the temporary nature of this exacerbation and encourage him/her to keep performing the exercises. The sessions will take place at the home of the participant. A physiotherapist, specialised in VR, will oversee that all participating physiotherapists adhere to the treatment protocol. We will conduct a process evaluation with the physiotherapists after the trial is completed.

\section{Usual care (trial arm 3)}

All included patients of trial arm $3(n=100)$ will receive usual care by their GPs. After randomisation, they will be redirected to a web page within the intervention that provides more information about the content of their trial arm. They will not receive access to online VR at this time, but will get access after the trial is completed. It is emphasised that their cooperation in the trial is vital for the success of the trial. They are asked to $\log$ in to fill out the follow-up questionnaires after 3 months and 6 months. Participating GPs will receive a written instruction, asking them to diagnose causes of dizziness according to the Dutch guideline on dizziness ${ }^{31}$ and to treat identified disorders according to the guidelines of the Dutch College of GPs. ${ }^{31}$

\section{Adverse events}

VR rarely causes adverse events. Previous trials with the validated VR booklet did not report any serious adverse events related to dizziness or the intervention. ${ }^{14} 1720 \mathrm{~A}$ small proportion of patients reported minor, transient side effects like neck pain, feelings of nausea and an increase in dizziness. Recently, the results of the trial by Yardley et al $(\mathrm{n}=296)$ have illustrated the safety of internet-based VR (Geraghty et al personal communication). Eighteen hospitalisations were identified in a GP notes review undertaken at the end of the trial, 8 in the usual care arm and 10 in the intervention arm. None of these hospitalisations were related to dizziness. No dizziness/intervention-related serious adverse reactions were reported. Our version of the intervention will be a close Dutch translation of the British online VR intervention. Patients will be informed that minor side effects like a temporary increase of neck pain, nausea or dizziness may occur. They will be advised that these sensations are likely to disappear within 2 weeks by performing the exercises more slowly. All adverse events that occur to a participant during this study, whether or not considered related to VR, will be recorded. The Medical Ethical Committee of the VU Medical Center will be notified within 15 days in the case of the occurrence of a serious adverse event during the study. We will inform the participants and the Medical Ethical Committee if anything occurs, on the basis of which it appears that the disadvantages of participation may be significantly greater than that was foreseen in the research proposal. The study will then be suspended pending further review by the Medical Ethical Committee. Serious medical complications as a result of our VR intervention are very unlikely. Previous trials have generated a considerable body of evidence for the safety of VR ${ }^{13141720}$ (Geraghty et al personal communication). 
Data collection and storage

The Dutch intervention platform will be created using the LifeGuide software (https://www.lifeguideonline. org). LifeGuide is a set of open source software tools, which allows intervention designers to create interactive web-based interventions. The University of Southampton developed the LifeGuide software in 2008. Since then, numerous successful internet-based intervention trials using LifeGuide have been performed and published in high-impact scientific journals. These trials include public health interventions (eg, smoking cessation) ${ }^{32}$ and illness management interventions. ${ }^{33}$ 34 The LifeGuide software not only allows the user to create the intervention but also contains a data management system. LifeGuide collects output data on participant use and outcomes, stores it securely and provides facilities for collating and providing anonymised data. ${ }^{21}$ Patients will fill out a questionnaire at baseline, 3 months follow-up and 6 months follow-up. By using the LifeGuide software, we will also automatically (and nonintrusively) collect data on all aspects of intervention use, including which sections and pages are viewed, how often and for how long. We will use participant identification numbers and the principal investigator will safeguard the key to the code of the participant identification number. Only the principal investigator and the research assistants will have access to the source data. Data will be stored on the LifeGuide servers in the UK. These servers are protected by the firewall of the University of Southampton, in accordance with the data protection policy of this institution. Researchers from the VU Medical Center and the University of Southampton signed a data processing agreement. Data will be kept for as long as the study takes, and at least 15 years after completion. Handling of the data complies with the Dutch Personal Data Protection Act.

\section{Measures}

A list of all measures and the moment at which they are collected is displayed in table 1 .

\section{Demographic characteristics}

Patients are asked to complete seven demographic questions regarding age, gender, living situation, comorbidities and level of education and five questions about the nature of their dizziness symptoms at baseline. These questions are designed by the research team and are based on previously used baseline measures. ${ }^{14} 1720$

\section{Primary outcomes}

The primary outcome measure will be dizziness symptoms at 6 months. The VSS-SF, ${ }^{13} 35$ a short-form version of the VSS ${ }^{36}$ will be used to measure this. It is based on the frequency of 15 dizziness-related symptoms during the past month on a scale from 0 (no symptoms) to 4 (symptoms most days). Improvement can reflect either fewer or less frequent symptoms. The VSS-SF measures a broad concept of dizziness for which construct validity
Table 1 Outcome measures and times of collection within the trial

\begin{tabular}{llll}
\hline Measure & Baseline & $\begin{array}{l}\text { 3-month } \\
\text { follow-up }\end{array}$ & $\begin{array}{l}\text { 6-month } \\
\text { follow-up }\end{array}$ \\
\hline Demographics & $\mathrm{X}$ & & \\
VSS-SF & $\mathrm{X}$ & $\mathrm{X}$ & $\mathrm{X}$ \\
iMCQ & $\mathrm{X}$ & $\mathrm{X}$ & $\mathrm{X}$ \\
iPCQ & $\mathrm{X}$ & $\mathrm{X}$ & $\mathrm{X}$ \\
EQ-5D-5L & $\mathrm{X}$ & $\mathrm{X}$ & $\mathrm{X}$ \\
DHI & $\mathrm{X}$ & $\mathrm{X}$ & $\mathrm{X}$ \\
SI & & $\mathrm{X}$ & $\mathrm{X}$ \\
PHQ & $\mathrm{X}$ & $\mathrm{X}$ & $\mathrm{X}$ \\
PETS & & $\mathrm{X}$ & $\mathrm{X}$ \\
\hline
\end{tabular}

DHI, Dizziness Handicap Inventory; EQ-5D-5L, 5-level EuroQol 5-dimension questionnaire; iMCQ, iMTA Medical Consumption Questionnaire; iPCQ, iMTA Productivity Cost Questionnaire; PETS, Problematic Experiences of Therapy Scale; PHQ, PRIME-MD Patient Health Questionnaire; SI, Subjective Improvement in Dizziness Symptoms; VSS-SF, Vertigo Symptoms Scale-Short Form.

has been demonstrated. It has been used effectively in many previous VR trials ${ }^{14} 1720$ (Geraghty et al personal communication), and demonstrated excellent discriminative ability (area under the curve (AUC) 0.87), high internal consistency (Cronbach's $\alpha 0.90$ ) and high testretest reliability (intraclass correlation coefficient (ICC) $0.88) .{ }^{37}$

\section{Health economic outcome measures}

Costs will be measured from a societal perspective according to the Dutch guidelines for economic evaluations $^{38}$ using internet questionnaires based on the iMTA Medical Consumption Questionnaire (iMCQ) ${ }^{39}$ and the iMTA Productivity Cost Questionnaire (iPCQ) ${ }^{40}$ at baseline, and after 3 and 6 months of follow-up. Healthcare costs include costs of primary and secondary care, complementary care and home care. Productivity costs include absenteeism from paid and unpaid work, and presenteeism (being present at work but not being able to work to one's full potential due to health symptoms). The friction cost approach will be used to estimate lost productivity costs. For the valuation of healthcare utilisation, standard prices published in the Dutch costing guidelines will be used. Medication use will be valued using prices of the Royal Dutch Society for Pharmacy. ${ }^{41}$ Quality of life will be evaluated using the 5-level EuroQol 5-dimension questionnaire (EQ-5D-5L) ${ }^{42}$ at baseline, and after 3 and 6 months of follow-up. The EQ-5D-5L is a self-report questionnaire consisting of five dimensions on which respondents indicate their own health state. The Dutch EQ-5D tariff will be used to calculate Quality-Adjusted Life-Years (QALYs).

\section{Secondary outcomes}

\section{Dizziness-related impairment}

We will assess dizziness-related impairment by using the Dizziness Handicap Inventory (DHI) at baseline, and 
after 3 and 6 months of follow-up. The DHI has 25 items with three answer categories ('yes', 'sometimes' or 'no'), investigating self-perceived physical, emotional and functional disability associated with dizziness. DHI scores range from 0 to 100 . A higher DHI score indicates a higher level of dizziness-related impairment. ${ }^{43}$ The DHI is a widely used self-report questionnaire, designed to quantify the impact of dizziness on everyday life. Construct validity exists for the DHI as a reliable indicator of dizziness-related impairment. ${ }^{44}$ The DHI has high internal consistency (Cronbach's $\alpha$ 0.89) and excellent test-retest reliability (ICC 0.97). ${ }^{43}$

\section{Subjective improvement in dizziness}

The subjective improvement in dizziness symptoms will be investigated by asking participants to report improvement or no change/worsening in subjective experience of dizziness (dichotomous) after 3 and 6 months of follow-up. ${ }^{13}$

\section{Anxiety and depression}

The presence or absence of anxiety and/or depression -at baseline, and after 3 and 6 months of follow-upwill be assessed using the PRIME-MD Patient Health Questionnaire (PHQ) ${ }^{45}$ an instrument to assess psychiatric disorders. This is a self-report questionnaire, which consists of 59 questions and enquires about recently experienced psychiatric symptoms. Using the PHQ algorithm, we will determine for each patient the presence or absence of the diagnoses Panic Disorder, Other Anxiety Disorder and Major Depressive Disorder at baseline. The diagnostic algorithms of the PHQ have been shown to have good criterion validity with reference to gold standard measures of mental disorders. Patients might experience these questions about their mental health as invasive to their privacy. We will assure the patient that all collected information is strictly confidential and will be anonymised.

\section{Process evaluation outcome measures \\ Quantitative data}

The Problematic Experiences of Therapy Scale (PETS) ${ }^{46}$ will be used to compare adherence to treatment in trial arm 1 (stand-alone internet-based VR) and trial arm 2 (blended care) after 3 and 6 months of follow-up. Patients in trial arm 3 do not have to fill out the PETS. The PETS is a brief measure to assess adherence levels and self-reported perceived barriers to adherence to physical rehabilitative therapy. It consists of 12 questions within 5 domains, that is, 'symptoms too severe or aggravated by therapy', 'uncertainty about how to carry out the treatment', 'doubts about treatment efficacy', 'practical problems' and 'lack of support'. These questions are then followed by intervention-specific questions about actual adherence levels to elements of the intervention; the questions to be used in this study have been used in previous studies of booklet-based VR. ${ }^{14}{ }^{20}$ Engagement of the digital intervention will be measured by automatically collected detailed data by the LifeGuide software with regard to intervention usage, number of logins, page views and time spent on each page. We will send a survey to all participating GPs and physiotherapists after the study is concluded to provide more insights in the views of the healthcare professionals involved in the intervention. Finally, we will contact patients who decided to discontinue the trial by telephone to assess their reason(s) for quitting.

\section{Qualitative data}

On their completion of the intervention, we will perform semistructured face-to-face interviews among a sample of 20-30 participants, 10-15 participants from trial arm 1 (stand-alone internet-based VR) and 10-15 participants from trial arm 2 (internet-based VR with physiotherapeutic support), respectively. The focus of these interviews is to learn about the patient's experiences of the VR intervention over the past weeks. The questions are about participants' existing management of their dizziness, their expectations about the VR intervention and initial experiences of engaging with it, any features they have found especially difficult or especially helpful, concerns they might have had about continuing and what they feel they had learnt. All interviews will be audio-recorded and transcribed verbatim.

Box 1 gives an overview of all measures and times of collection within the trial.

\section{Statistical analysis \\ Effectiveness intervention}

General characteristics of all participants will be described quantitatively. The data derived from questionnaires will also be presented in a quantitative way. Missing data will be handled with multiple imputation analysis. ${ }^{47}$ For the effectiveness analysis, we will perform intention-to-treat and per-protocol analyses. Patients in trial arms 1 and 2 that have completed all 6 weekly online sessions will be used for the per-protocol analysis. We will use multiple linear regression analysis to compare the primary outcome measure (VSS-SF) in participants allocated to trial arm 1 (stand-alone internetbased VR) and participants allocated to trial arm 2 (internet-based VR with physiotherapeutic support) with participants allocated to trial arm 3 (usual care). We will adjust for dizziness severity at baseline (the stratification variable) and any other potential confounders.

\section{Cost-effectiveness intervention}

For the cost-effectiveness analysis, ${ }^{48}$ we will perform costeffectiveness and cost-utility analyses with a time horizon of 6 months, making discounting unnecessary. Societal costs will be related to the primary outcome of the trial and QALYs in the economic evaluation. The analysis will be performed according to the intention-to-treat principle. Missing cost and effect data will be imputed using multiple imputations. Differences in costs and effects will be estimated using bivariate regression models while 
adjusting for confounders if necessary. Incremental costeffectiveness ratios (ICERs) will be calculated by dividing the difference in mean total costs between the treatment groups by the difference in mean effects. Bootstrapping with 5000 replications will be used to estimate $95 \%$ CIs around cost differences and the uncertainty surrounding the ICERs. Uncertainty surrounding the ICERs will be graphically presented on cost-effectiveness planes. Cost-effectiveness acceptability curves showing the probability that the intervention is cost-effective in comparison with usual care for a range of different ceiling ratios will also be estimated.

\section{Prediction model}

We will perform an additional analysis on the data to identify predictors of successful treatment in patients with dizziness for all forms of treatment. Secondary outcome measures will be analysed using linear regression for continuous outcomes and logistic regression for dichotomous outcomes, adjusting for dizziness severity at baseline and any other potential confounders. According to Moons et al, ${ }^{49}$ we will develop three prediction models: (1) predictors of successful treatment with internet-based VR (population: trial arms 1 and 2; $\mathrm{N}=200$ ), (2) predictors of successful treatment with stand-alone internet-based VR (population: trial arm 1; $\mathrm{N}=100$ ) and (3) predictors of successful treatment with internet-based VR with physiotherapeutic support (population: trial arm 2; $\mathrm{N}=100$ ). We will perform multiple logistic regression analysis with 'successful treatment after six months' (yes/no) as a dependent variable. Successful treatment will be defined as: (1) improvement in the VSS-SF score with at least three points (clinically significant change), ${ }^{8}$ (2) improvement in the DHI score with at least 11 points (clinically significant change $)^{44}$ and (3) subjective improvement in dizziness symptoms. ${ }^{13}$ Candidate predictors will be easy obtainable patient characteristics, for example, age, gender, education and dizziness characteristics. Prior to regression analysis, we will perform univariate logistic regression analysis to investigate the associations between separate candidate predictors and the dependent variable. To assess the reliability of the model, we will calculate the Hosmer-Lemeshow goodness-of-fit statistic and construct calibration plots. To assess the discriminative ability of the model, we will calculate the AUC. Since prediction models perform better in development cohorts than in other similar populations (overfitting), we will perform a bootstrapping procedure. During this procedure, we will repeat the entire modelling process, in order to validate the final model and to adjust (shrink) the estimated performance and regression coefficients.

\section{Process evaluation analysis}

Analysis of quantitative data

The PETS, the LifeGuide software usage data, the survey results of participating GPs and physiotherapists in the trial and reasons for discontinuing the trial will all be analysed using descriptive statistics.

\section{Analysis of qualitative data}

Thematic analysis will be employed to identify patterns within the transcribed face-to-face interviews. Two researchers will independently read and re-read the first interviews to gain an overall impression and generate a list of initial codes. This list will be discussed within the research group, in order to reach consensus on each code and to categorise the identified codes into themes. Once a provisional coding scheme is developed, two independent researchers will code the other interviews. Afterwards, the coding results of each interview will be discussed until consensus is reached. The results of the qualitative study will be used to provide insights into (1) patients' experiences during the intervention and (2) possible reasons for outcome and adherence results.

\section{DISSEMINATION}

An independent monitor from the Clinical Research Bureau (CRB) of VUmc will have access to the data and source documents of the trial. Monitoring will be performed in compliance with Good Clinical Practice and other rules and regulations in order to achieve highquality research and secure patient safety.

We aim to widely disseminate the insights and results of this project to (national and international) GPs, GP trainees, elderly care physicians, elderly care physician trainees, physiotherapists and patients with dizziness. We will publish the research results in international peerreviewed journals. There are no restrictions placed on publication by the sponsor of this study (ZonMw, programma Kwaliteit van Zorg: Versnellen, verbreden, vernieuwen). If proven effective, we plan to make the intervention available to all GPs in the Netherlands. The website of http://www.thuisarts.nl has been developed by the Dutch College of GPs in 2011 in order to offer patients reliable medical information and to support GPs. ${ }^{50}$ This site is increasingly visited by patients (6.2 million visitors in first half of 2014) and will function as an important resource to inform patients with dizziness. $^{50}$ Finally, we will distribute our results by means of (national and international) conference presentations. Up to now, other medical specialties in the field of dizziness, like ENT and neurology, are hardly aware of the growing expertise on dizziness within the discipline of general practice. Therefore, we also aim to disseminate the insights and results of this project to other relevant medical specialties in the field of dizziness.

\section{DISCUSSION}

\section{Introducing blended care to treat dizziness}

Research on the effectiveness of the combination of online and face-to-face therapy (blended care) is scarce and has never been investigated in patients with vertigo. 
Stand-alone online interventions are susceptible to nonadherence and attrition. Blended care has been shown to be able to address these issues. ${ }^{25}$ For instance, fear of exacerbation of symptoms may be an important reason for patients to discontinue their treatment. The encouragement and guidance of an experienced physiotherapist at this time may give patients the confidence to keep doing the exercises.

\section{Providing patients with personalised care}

Nowadays, interventions can be tailored to the individual needs of the patient. Tailored care is created by the online intervention by continuously re-evaluating the intensity of the exercises for each patient. Different patients (with regard to age, gender, education and dizziness characteristics) may also have different preferences. We will attempt to develop a clinical decision rule for predictors of successful treatment to ensure personalised treatment. With a decision rule, GPs will be able to discriminate between patients who are likely to benefit from stand-alone internet-based VR and those who need a combination of online and face-to-face therapy (blended care). The intervention may not only reduce dizziness and dizziness-related impairment, but also stimulate patients' self-management, in line with the current policy on medical care.

\section{Increasing treatment options for GPs and reducing healthcare costs}

The availability of an effective online intervention will offer a cost-effective alternative for treating dizziness, as costly referrals can be avoided. It may stimulate GPs to reduce inappropriate drug prescribing. According to the Second Dutch National Survey of General Practice, GPs prescribed ineffective drugs to as much as $10 \%$ of older patients with dizziness during the first consultation. ${ }^{51}$ Moreover, adverse consequences of dizziness—such as falls, anxiety and depression-will be reduced, leading to less healthcare usage and healthcare costs. From a wider perspective, the project has the capacity to illustrate how easily accessible interventions in primary care may relieve the burden on secondary/tertiary care, leading to better care at lower costs. ${ }^{52}$

Contributors VAvV wrote the manuscript. ORM, JCvdW, JEB, MS, WvD, RE, $\mathrm{LY}$ and $\mathrm{HEvdH}$ were involved in creating the study design and protocol. RE and $L Y$ also gave a valuable input based on previous experience with vestibular rehabilitation trials. ORM is the coordinator of the study and obtained the grant.

Funding This study was funded by ZonMw (programma Kwaliteit van Zorg: Versnellen, verbreden, vernieuwen; grant number 839110015). The results of the study will be submitted for publication to peer-reviewed scientific journals. There are no restrictions placed on publication by ZonMw.

\section{Competing interests None declared.}

Ethics approval The institutional review board of VU University Medical Center.

Provenance and peer review Not commissioned; peer reviewed for ethical and funding approval prior to submission.
Open Access This is an Open Access article distributed in accordance with the Creative Commons Attribution Non Commercial (CC BY-NC 4.0) license, which permits others to distribute, remix, adapt, build upon this work noncommercially, and license their derivative works on different terms, provided the original work is properly cited and the use is non-commercial. See: http:// creativecommons.org/licenses/by-nc/4.0/

\section{REFERENCES}

1. Bailey KE, Sloane PD, Mitchell M, et al. Which primary care patients with dizziness will develop persistent impairment? Arch Fam Med 1993:2:847-52.

2. Yardley L, Owen N, Nazareth I, et al. Prevalence and presentation of dizziness in a general practice community sample of working age people. Br J Gen Pract 1998;48:1131-5.

3. Hannaford PC, Simpson JA, Bisset AF, et al. The prevalence of ear, nose and throat problems in the community: results from a national cross-sectional postal survey in Scotland. Fam Pract 2005;22:227-33.

4. Benecke H, Agus S, Kuessner D, et al. The burden and impact of vertigo: findings from the REVERT patient registry. Front Neurol 2013;4:136.

5. Jonsson R, Sixt E, Landahl S, et al. Prevalence of dizziness and vertigo in an urban elderly population. J Vestib Res 2004;14:47-52.

6. Neuhauser HK, Radtke A, von Brevern $\mathrm{M}$, et al. Burden of dizziness and vertigo in the community. Arch Intern Med 2008;168:2118-24.

7. Dros J, Maarsingh OR, Beem L, et al. Functional prognosis of dizziness in older adults in primary care: a prospective cohort study. J Am Geriatr Soc 2012;60:2263-9.

8. Kroenke K, Lucas CA, Rosenberg ML, et al. Causes of persistent dizziness. A prospective study of 100 patients in ambulatory care. Ann Intern Med 1992;117:898-904.

9. Sloane PD, Dallara J, Roach C, et al. Management of dizziness in primary care. J Am Board Fam Pract 1994;7:1-8.

10. Hanley K, O' Dowd T. Symptoms of vertigo in general practice: a prospective study of diagnosis. Br J Gen Pract 2002;52:809-12.

11. Jayarajan V, Rajenderkumar D. A survey of dizziness management in General Practice. J Laryngol Otol 2003;117:599-604.

12. Rascol O, Hain TC, Brefel C, et al. Antivertigo medications and drug-induced vertigo. A pharmacological review. Drugs 1995;50:777-91.

13. McDonnell MN, Hillier SL. Vestibular rehabilitation for unilateral peripheral vestibular dysfunction. Cochrane Database Syst Rev 2015;CD005397.

14. Yardley L, Barker F, Muller I, et al. Clinical and cost effectiveness of booklet based vestibular rehabilitation for chronic dizziness in primary care: single blind, parallel group, pragmatic, randomised controlled trial. Br Med J 2012;344:e2237.

15. Brandt T. Management of vestibular disorders. J Neurol 2000;247:491-9.

16. Beidel DC, Horak FB. Behavior therapy for vestibular rehabilitation $J$ Anxiety Disord 2001:15:121-30.

17. Yardley L, Donovan-Hall M, Smith HE, et al. Effectiveness of primary care-based vestibular rehabilitation for chronic dizziness. Ann Intern Med 2004;141:598-605.

18. Geraghty AW, Kirby S, Essery R, et al. Internet-based vestibular rehabilitation for adults aged 50 years and over: a protocol for a randomised controlled trial. BMJ Open 2014;4:e005871.

19. Van Vugt VA, Diaz Nerio PM, Van der Wouden JC, et al. Use of canalith repositioning manoeuvres and vestibular rehabilitation: a GP survey. Scand J Prim Health Care 2017, in press.

20. Yardley L, Kirby S. Evaluation of booklet-based self-management of symptoms in Meniere disease: a randomized controlled trial. Psychosom Med 2006;68:762-9.

21. Williams S, Yardley L, Wills GB. A qualitative case study of LifeGuide: users' experiences of software for developing internet-based behaviour change interventions. Health Informatics $J$ 2013;19:61-75.

22. https://www.cbs.nl/nl-nl/nieuws/2013/50/internetgebruik-ouderenfors-toegenomen (30 November 2016).

23. Aalbers T, Baars MA, Rikkert MG. Characteristics of effective Internet-mediated interventions to change lifestyle in people aged 50 and older: a systematic review. Ageing Res Rev 2011;10: 487-97.

24. Wilhelmsen M, Lillevoll K, Risor MB, et al. Motivation to persist with internet-based cognitive behavioural treatment using blended care: a qualitative study. BMC Psychiatry 2013;13:296.

25. van Ballegooijen $W$, Cuijpers $P$, van Straten $A$, et al. Adherence to internet-based and face-to-face cognitive behavioural therapy for depression: a meta-analysis. PLOS ONE 2014;9:e100674. 
26. van der Vaart $\mathrm{R}$, Witting $\mathrm{M}$, Riper $\mathrm{H}$, et al. Blending online therapy into regular face-to-face therapy for depression: content, ratio and preconditions according to patients and therapists using a Delphi study. BMC Psychiatry 2014;14:355

27. Maarsingh OR, Dros J, Schellevis FG, et al. Causes of persistent dizziness in elderly patients in primary care. Ann Fam Med 2010;8:196-205.

28. Wegner I, Niesten ME, van Werkhoven $\mathrm{CH}$, et al. Rapid systematic review of the Epley maneuver versus vestibular rehabilitation for benign paroxysmal positional vertigo. Otolaryngol Head Neck Surg 2014;151:201-7.

29. Essery R, Kirby S, Geraghty AW, et al. The development of balance retraining: an online intervention for dizziness in adults aged 50 years and older. Am J Audiol 2015;24:276-9.

30. Meli A, Zimatore G, Badaracco C, et al. Effects of vestibular rehabilitation therapy on emotional aspects in chronic vestibular patients. J Psychosom Res 2007;63:185-90.

31. van Lieshout J, Assendelft WJ, Nederlands Huisartsen G. [Summary of the Dutch College of General Practitioners' practice guideline 'Dizziness']. Ned Tijdschr Geneeskd 2003;147:331-5.

32. Brown J, Michie S, Geraghty AW, et al. Internet-based intervention for smoking cessation (StopAdvisor) in people with low and high socioeconomic status: a randomised controlled trial. Lancet Respir Med 2014;2:997-1006.

33. Little $\mathrm{P}$, Stuart B, Francis $\mathrm{N}$, et al. Effects of internet-based training on antibiotic prescribing rates for acute respiratory-tract infections: a multinational, cluster, randomised, factorial, controlled trial. Lancet 2013;382:1175-82

34. Little $\mathrm{P}$, Stuart B, Hobbs FD, et al. An internet-delivered handwashing intervention to modify influenza-like illness and respiratory infection transmission (PRIMIT): a primary care randomised trial. Lancet 2015;386:1631-9.

35. Yardley L, Burgneay J, Andersson G, et al. Feasibility and effectiveness of providing vestibular rehabilitation for dizzy patients in the community. Clin Otolaryngol Allied Sci 1998;23:442-8.

36. Yardley L, Masson E, Verschuur C, et al. Symptoms, anxiety and handicap in dizzy patients: development of the vertigo symptom scale. J Psychosom Res 1992;36:731-41.

37. Wilhelmsen K, Strand LI, Nordahl SH, et al. Psychometric properties of the Vertigo Symptom Scale-Short Form. BMC Ear Nose Throat Disord 2008;8:2.

38. Hakkaart-van Roijen L, Tan S, Bouwmans C. Handleiding voor kostenonderzoek [Dutch manual for costing in economic evaluations]. 2011. https://www.zorginstituutnederland.nl/binaries/ content/documents/zinl-www/documenten/publicaties/overigepublicaties/1602-richtlijn-voor-het-uitvoeren-van-economischeevaluaties-in-de-gezondheidszorg-bijlagen/Richtlijn+voor+het +uitvoeren+van+economische+evaluaties+in+de+gezondheidszorg +(verdiepingsmodules).pdf (30 November 2016).

39. Bouwmans C, Hakkaart-van Roijen L, Koopmanschap M, et al. Handleiding iMTA Medical Cost Questionnaire (iMCQ). 2013. www. imta.nl

40. Bouwmans $\mathrm{C}$, Krol M, Severens $\mathrm{H}$, et al. The iMTA productivity cost questionnaire: a standardized instrument for measuring and valuing health-related productivity losses. Value Health $2015 ; 18$ : 753-8.

41. https://www.z-index.nl/g-standaard (30 November 2016).

42. Herdman M, Gudex C, Lloyd A, et al. Development and preliminary testing of the new five-level version of EQ-5D (EQ-5D-5L). Qual Life Res 2011;20:1727-36.

43. Jacobson GP, Newman CW. The development of the Dizziness Handicap Inventory. Arch Otolaryngol Head Neck Surg 1990;116:424-7.

44. Tamber AL, Wilhelmsen KT, Strand LI. Measurement properties of the Dizziness Handicap Inventory by cross-sectional and longitudinal designs. Health Qual Life Outcomes 2009;7:101.

45. Spitzer RL, Kroenke K, Williams JBW, et al. Validation and utility of a self-report version of PRIME-MD - the PHQ primary care study. JAMA 1999;282:1737-44.

46. Kirby S, Yardley L, Donovan-Hall M. Measuring and predicting problematic experiences related to non-adherence in exercise based rehabilitation. Psychol Health 2012;27:69.

47. Donders AR, van der Heijden GJ, Stijnen T, et al. Review: a gentle introduction to imputation of missing values. J Clin Epidemiol 2006;59:1087-91.

48. Fenwick E, O’Brien BJ, Briggs A. Cost-effectiveness acceptability curves-facts, fallacies and frequently asked questions. Health Econ 2004:13:405-15.

49. Moons KG, Kengne AP, Woodward M, et al. Risk prediction models: I. Development, internal validation, and assessing the incremental value of a new (bio)marker. Heart 2012;98:683-90.

50. Drenthen T, Beijaert RP, Jansen PW, et al. [What do you think of Thuisarts.nl? Experiences after 3 years of www.Thuisarts.nl]. Ned Tijdschr Geneeskd 2014;158:A8282.

51. Maarsingh OR, Schellevis FG, van der Horst HE. Looks vestibular: irrational prescribing of antivertiginous drugs for older dizzy patients in general practice. Br J Gen Pract 2012;62:518-20.

52. Starfield B. Is primary care essential? Lancet 1994;344:1129-33. 